\title{
Intelligent Prediction Techniques for Chronic Kidney Disease Data Analysis
}

\author{
Shanmugarajeshwari V., Kalasalingam Academy of Research and Education, Krishnankoil, India \\ Ilayaraja M., Kalasalingam Academy of Research and Education, Krishnankoil, India
}

\begin{abstract}
Information is stored in various domains like finance, banking, hospital, education, etc. Nowadays, data stored in medical databases are growing rapidly. The proposed approach entails three parts comparable to preprocessing, attribute selection, and classification C5.0 algorithms. This work aims to design a machine-based diagnostic approach using various techniques. These algorithms improve the efficiency of mining risk factors of chronic kidney diseases, but there are also have some shortcomings. To overcome these issues and improve an effectual clinical decision support system exhausting classification methods over a large volume of the dataset for making better decisions and predictions, this paper presents grouping classification assembly through consuming the C5.0 algorithm, pointing towards assembling time to acquire great accuracy to identify an early diagnosis of chronic kidney disease patients with risk level by analyzing the chronic kidney disease dataset.
\end{abstract}

\section{KEYWORDS}

Artificial Intelligence, Chronic Kidney, Data Mining, Deep Learning Techniques, Intelligent Decision Support System, Machine Learning Techniques

\section{INTRODUCTION}

In data mining is an analyzing or discovering good knowledge to develop the meaningful collection of data from a huge amount of data using the knowledge. The health specifying care is the solicitation of information using machine learning algorithms. To developing also exploring healthcare data records analytical surroundings are using various methods to superior raise the value of health-related problem to prediction.

Health-care record data is mostly gorgeous derived from a worldwide diversity of foundations such as sensor devices, images, text in the system of automated electrical archives. In this miscellaneous in the collection of data and depiction method clues to several trials in together the handling process and analysis of the original data. World wide assortment in the methods is essential to evaluate dissimilar forms of records (Reddy \& Aggarwal, 2015).

The kidneys' operations are to pass through a filter of the blood. It eliminates unwanted blood to regulate the stability of electrolytes and fluid. It strains blood, they create urine, which two beanshaped structure of the kidney. Every one kidney surrounds a million things of unit so-called nephrons (Urinary Incontinence, n.d.).

\section{DOI: 10.4018/IJAIML.20210701.oa2}

This article, published as an Open Access article on April 23, 2021 in the gold Open Access journal, International Journal of Artificial Intelligence and Machine Learning (converted to gold Open Access on January 1, 2021), is distributed under the terms of the Creative Commons Attribution License (http://creativecommons.org/licenses/by/4.0/) which permits unrestricted use, distribution, and production in any medium, provided the author of the original work and original publication source are properly credited. 


\section{Factors of Chronic Kidney Disease}

The following are some of the factors which lead to chronic kidney disease, the main cause is diabetes and others are hypertension, smoke, fatness, heart illness, family record, alcohol, and age problem.

\section{Symptoms}

Some of the warning sign is listed down, that could be variations to urinary function, plasma in the urine, bulge \& pain, severe tiredness and weakness.

\section{Types: Acute and Chronic}

- Acute Prerenal Kidney Failure -Suddenly decreases blood flow.

- Acute Intrinsic Kidney Failure -Straight injury to the kidneys foundations unexpected damage in kidney.

- Chronic Prerenal Kidney Failure - Gradually decreases blood flow.

- Chronic Intrinsic Kidney Failure - Direct damage to the kidneys cause a gradual loss in kidney function (Bala \& Kumar, 2014).

Chronic Kidney Disease (CKD) is a worldwide health crisis. In 2019, the World Health Organization agree to fifty-eight million deaths and 35 million recognized to chronic kidney disease. The world level 850 million people now predicted to have kidney diseases from many causes, chronic kidney disease causes at least 2.4 million deaths world wide-reaching per year sixth fastest-growing cause of disease and death. Dialysis is a fashion of life for many patients pain with kidney sicknesses in India. The medical record of Government of TamilNadu, India, Every one year 2.2 Lakh fresh patients affected by final point renal disease or end-stage renal disease. According to the Global Burden of Disease (GBD) learning, kidney disease was hierarchical $27^{\text {th }} 1990$ but rose to $18^{\text {th }}$ in 2010 and $9^{\text {th }}$ in 2019. Motivations on the development and use of machine learning algorithms for classical methods using other machine learning approaches to achieve high accuracy.

Figure 1 represents the various factors are affecting the patient data are evaluated with healthcare data analytics.

\section{RELATED WORKS}

A Literature survey refers to a critical summary. Literature reviews contextualize research about a topic. A literature review is an evaluative report of studies found in the literature related to a selected area. The review should describe, summarize, evaluate and clarify this literature. It determines the

\section{Figure 1. Affecting factors of the Healthcare Data Analytics}

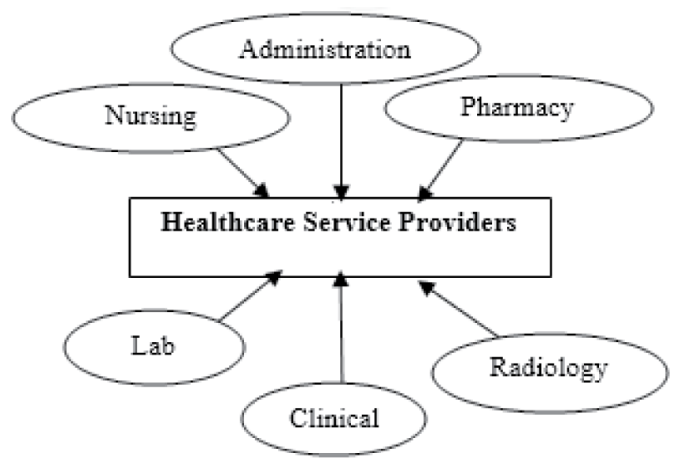


context of the research around a subject area. It is appraising explosion preparations start in the literature associated with a particular area. The journal should designate, review, estimate from the survey (Feature Selection, n.d.). It is a full theory base aimed at the study and benefits the researcher to the environment of the investigation. The analyses have been done on various topics of an outline. The root of the prevailing information, everybody to building advanced knowledge and thought for advance study perseverance (Queens U, n.d.) (Table 1).

\section{FINDINGS}

From this review, it is concrete that healthcare decision support clinical performance can be assessed by smearing, machine learning techniques can be valued by various algorithms. In this survey, our research work ordered as three parts. The best algorithm is deep learning to deal with huge datasets, using an $\mathrm{R}$ programming language is used. This research work presents an algorithm on the classification structure by various artificial intelligence and machine learning algorithms that have resulted in good accuracy. In the future, the proposed research work has been successfully implemented in R with the Graphical User Interface (GUI) environment.

\section{Overview of the Model}

The first objective is an early diagnosis of Chronic Kidney Disease (CKD) patients with risk levels by analyzing chronic kidney disease dataset. This objective plays a valuable role in current research since many patients suffer from this disease around the world (Figures 2-3).

\section{Phase 1: Preprocess}

- Dataset Depiction

- Cataloging

The second objective is the power of the feature selection using machine learning methods to detect the patients with the risk level of chronic kidney diseases while affected by particular symptoms of a particular disease (Figures 4-9).

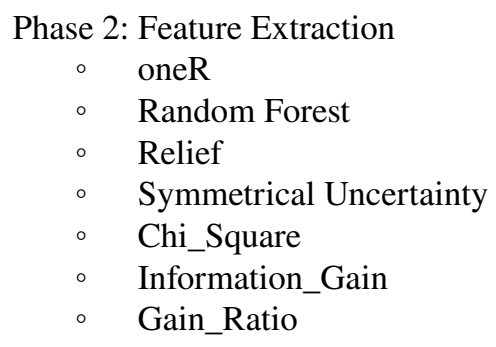

\section{Attribute Selection}

It is likewise a feature selection (HKU, n.d.). Now, choosing to apply features and neglect the inappropriate attributes. These methods were applied to the preprocessed data set which has 4050 samples, concentrated on picking out all attributes. The greatest attribute variety technique is to gain ratio feature selection that has been functional to the preprocessed records (Celik et al., 2014).

\section{Gain Ratio Feature Selection}

It is attributed variety technique to gain ratio with the attribute method. The feature selection method is used to extract the relevant features and discard the irrelevant features. This method applied for chronic kidney disease datasets (Celik et al., 2014): 
Table 1. Literature Review for Analysis of Chronic Kidney Disease

\begin{tabular}{|c|c|c|c|c|c|}
\hline Author & Problem & Software & & hniques & $\begin{array}{c}\text { Accuracy } \\
\text { Performance (\%) }\end{array}$ \\
\hline \multirow{10}{*}{ Dowluru, et al. (2012) } & \multirow{10}{*}{$\begin{array}{l}\text { Analysis of Kidney } \\
\text { stone }\end{array}$} & \multirow{4}{*}{ WEKA Tool } & \multicolumn{2}{|c|}{ Naive Bayes Classification } & 0.99 \\
\hline & & & \multicolumn{2}{|c|}{ Logistic Regression } & 1.00 \\
\hline & & & \multicolumn{2}{|c|}{ J48 Algorithm } & 0.97 \\
\hline & & & \multicolumn{2}{|c|}{ Random Forest } & 0.98 \\
\hline & & & \multicolumn{2}{|c|}{ Naive Bayes } & 0.79 \\
\hline & & & \multicolumn{2}{|l|}{ K-NN } & 0.7377 \\
\hline & & & \multicolumn{2}{|c|}{ Classification tree } & 0.9352 \\
\hline & & 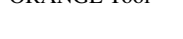 & \multicolumn{2}{|l|}{$\mathrm{C} 4.5$} & 0.9352 \\
\hline & & & \multicolumn{2}{|l|}{ SVM } & 0.9198 \\
\hline & & & \multicolumn{2}{|c|}{ Random Forest } & 0.9352 \\
\hline \multirow{3}{*}{ Lakshmi, et al. (2014) } & \multirow{3}{*}{$\begin{array}{l}\text { Problem with Kidney } \\
\text { dialysis }\end{array}$} & \multirow{3}{*}{ TANAGRA } & \multicolumn{2}{|l|}{ ANN } & 93.852 \\
\hline & & & \multicolumn{2}{|c|}{ Decision Tree(C5) } & 78.4455 \\
\hline & & & \multicolumn{2}{|c|}{ Logical Regression } & 74.7438 \\
\hline \multirow{2}{*}{ Eyck, et al. (2012) } & \multirow{2}{*}{ A.K.I } & \multirow{2}{*}{ matlab } & \multicolumn{2}{|c|}{ Gaussian-aROC } & 0.758 \\
\hline & & & \multicolumn{2}{|c|}{ Gussian- RMSER } & 0.408 \\
\hline \multirow{2}{*}{ Zadeh, et al. (2013) } & \multirow{2}{*}{ Early AVF Failure } & \multirow{2}{*}{ WEKA Tool } & \multicolumn{2}{|c|}{ W-Simple Cart } & 85.11 \\
\hline & & & WJ48 & & 80.85 \\
\hline $\begin{array}{l}\text { Abeer Y. Al- Hyari, et al. } \\
\text { (2012) }\end{array}$ & $\begin{array}{l}\text { Enduring Kidney } \\
\text { disease }\end{array}$ & WEKA Tool & Decisic & & -- \\
\hline Song et al. (2012) & $\begin{array}{l}\text { Renal failure } \\
\text { Hemodialysis }\end{array}$ & WEKA Tool & Decisic & & $60-80$ \\
\hline Sriraam, et al. (2006) & $\begin{array}{l}\text { Treatment of Kidney } \\
\text { Dialysis }\end{array}$ & WEKA Tool & Associ & Rule & 97.7 \\
\hline Jain, et al. (2014) & $\begin{array}{l}\text { Nephrotic } \\
\text { syndrome(total protein) }\end{array}$ & TANAGRA Tool & $\mathrm{C} 4.5$ & & $\begin{array}{l}11 \\
\text { (error rate) }\end{array}$ \\
\hline Joce ol (2012) & Analysis of Kidney & MATI A P & Associ & Rule & 02 \\
\hline (2) & Image & 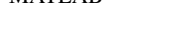 & Navie 1 & & 然 \\
\hline & & & & MLP & 0.9613 \\
\hline Kumar, et al. (2012) & $\begin{array}{l}\text { Kidney Stone treatment } \\
\text { and symptoms }\end{array}$ & WEKA & ANN & LVQ & 0.8459 \\
\hline & & & & RBF & 0.8732 \\
\hline & & & SVM & & 0.91 \\
\hline & & & PLS & & 0.83 \\
\hline & & & FFNN & & 0.85 \\
\hline Leung, et al. (2013) & $\begin{array}{l}\text { Danger forms in } \\
\text { diabetic kidney disease }\end{array}$ & MATLAB & RPART & & 0.87 \\
\hline & & & Randor & & 0.91 \\
\hline & & & Naïve 1 & & 0.86 \\
\hline & & & C5.0 & & 0.90 \\
\hline Bala, et al. (2014) & $\begin{array}{l}\text { Review of Kidney } \\
\text { Disease Prediction }\end{array}$ & - & - & & - \\
\hline Viigurani et ol (2015) & Kidney Disease & $\mathrm{MATI} A \mathrm{P}$ & SVM & & 76.32 \\
\hline 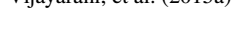 & Prediction & 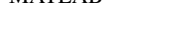 & ANN & & 87.70 \\
\hline
\end{tabular}


Table 1. Continued

\begin{tabular}{|c|c|c|c|c|}
\hline Author & Problem & Software & Techniques & $\begin{array}{c}\text { Accuracy } \\
\text { Performance (\%) }\end{array}$ \\
\hline \multirow{2}{*}{ Vijayarani, et al. (2015b) } & \multirow{2}{*}{$\begin{array}{l}\text { Arrangement Processes } \\
\text { for Kidney Ailment } \\
\text { Forecast }\end{array}$} & \multirow{2}{*}{ MATLAB } & SVM & 76.32 \\
\hline & & & Naïve Bayes & 70.96 \\
\hline \multirow{2}{*}{ Sinha, et al. (2015) } & \multirow{2}{*}{$\begin{array}{l}\text { Learning of Lasting } \\
\text { Kidney Virus } \\
\text { Expectation }\end{array}$} & \multirow{2}{*}{ MATLAB } & SVM & 0.7375 \\
\hline & & & $\mathrm{KNN}$ & 0.7875 \\
\hline \multirow{6}{*}{ Jena, et al. (2015) } & \multirow{6}{*}{$\begin{array}{l}\text { Guess of Chronic- } \\
\text { Kidney-Disease }\end{array}$} & \multirow{6}{*}{ WEKA } & Naïve Bayes & 95 \\
\hline & & & Multilayer Perceptron & 99.75 \\
\hline & & & SVM & 62 \\
\hline & & & J48 & 99 \\
\hline & & & Conjuctive Rule & 94.75 \\
\hline & & & Decision Table & 99 \\
\hline \multirow{3}{*}{ Ramya, et al. (2016) } & \multirow{3}{*}{$\begin{array}{l}\text { Judgment of Chronic } \\
\text { Kidney Disease }\end{array}$} & \multirow{3}{*}{$\mathrm{R}$} & $\mathrm{BP}$ & 80.4 \\
\hline & & & RBF & 85.3 \\
\hline & & & Random Forest (RF) & 78.6 \\
\hline \multirow{2}{*}{ Celik, et al. (2014) } & \multirow{2}{*}{$\begin{array}{l}\text { Diagnosis besides } \\
\text { Estimate of Fleeting } \\
\text { Kidney Disease }\end{array}$} & \multirow{2}{*}{ WEKA } & SVM & 97.06 \\
\hline & & & Decision Tree & 96.12 \\
\hline Norouzi, et al. (2016) & $\begin{array}{l}\text { Renal Miscarriage } \\
\text { Problem in CKD }\end{array}$ & MATLAB & ANFIS & 95 \\
\hline \multirow{5}{*}{ Sharma, et al. (2016) } & \multirow{5}{*}{$\begin{array}{l}\text { Chronic Kidney } \\
\text { Disease Diagnosis }\end{array}$} & \multirow{5}{*}{ MATLAB } & Decision Tree & 98.60 \\
\hline & & & SVM & 90.50 \\
\hline & & & ANN - MLFFNN & 88.50 \\
\hline & & & KNN & 88.88 \\
\hline & & & Discriminant Analysis & 90.80 \\
\hline \multirow{6}{*}{ Kumar, M., (2016) } & \multirow{6}{*}{$\begin{array}{l}\text { Prediction of Chronic } \\
\text { Kidney Disease }\end{array}$} & \multirow{6}{*}{ MATLAB } & $\mathrm{RF}$ & 95.67 \\
\hline & & & SMO & 90 \\
\hline & & & Naïve Bayes & 87.64 \\
\hline & & & $\mathrm{RBF}$ & 83.78 \\
\hline & & & MLPC & 89 \\
\hline & & & SLG & 87 \\
\hline \multirow{3}{*}{ Chatterjee, et al. (2017) } & \multirow{3}{*}{$\begin{array}{l}\text { Chronic Kidney } \\
\text { Disease Classification }\end{array}$} & \multirow{3}{*}{ MATLAB } & MLP-FFN & 96.33 \\
\hline & & & PSO-NN & 98.5 \\
\hline & & & NN-MCS & 99.6 \\
\hline \multirow{6}{*}{ Subhashini, et al. (2017) } & \multirow{6}{*}{$\begin{array}{l}\text { Performance analysis } \\
\text { of Chronic Kidney } \\
\text { Disease }\end{array}$} & \multirow{6}{*}{ MATLAB } & ANN & 93 \\
\hline & & & $\mathrm{KNN}$ & 96.76 \\
\hline & & & S_V_M & 87 \\
\hline & & & Naïve_Bayes & 88.9 \\
\hline & & & Decision_Tree & 86 \\
\hline & & & $\begin{array}{l}\text { Fuzzy_Ambiguous_ } \\
\text { Classifier }\end{array}$ & 90 \\
\hline
\end{tabular}


Table 1. Continued

\begin{tabular}{|c|c|c|c|c|}
\hline Author & Problem & Software & Techniques & $\begin{array}{c}\text { Accuracy } \\
\text { Performance (\%) }\end{array}$ \\
\hline \multirow{6}{*}{ Alasker, et al. (2017) } & \multirow{6}{*}{$\begin{array}{l}\text { Detection of Kidney } \\
\text { Disease }\end{array}$} & \multirow{6}{*}{ WEKA } & ANN & 99.5100 \\
\hline & & & Naïve Bayes & 99.5100 \\
\hline & & & Decision Table & 97.619 \\
\hline & & & $\mathrm{J} 48$ & 98.4127 \\
\hline & & & One R & 99.2063 \\
\hline & & & KNN & 97.619 \\
\hline Mahdavi-mazdeh, et al. (2018) & $\begin{array}{l}\text { Predict chronic kidney } \\
\text { disease progression }\end{array}$ & MATLAB & ANFIS & 98 \\
\hline \multirow{2}{*}{ Lakshmanaprabu, et al. (2019) } & \multirow{2}{*}{$\begin{array}{l}\text { Medical decision } \\
\text { support system }\end{array}$} & \multirow{2}{*}{ MATLAB } & DNN & 98.25 \\
\hline & & & PSO & 99.25 \\
\hline \multirow{11}{*}{ Pasadana, et al. (2019) } & \multirow{11}{*}{$\begin{array}{l}\text { Chronic Kidney } \\
\text { Disease Prediction }\end{array}$} & \multirow{11}{*}{ MATLAB } & Decision_Stump & 92 \\
\hline & & & Hoeffding_Tree & 95.75 \\
\hline & & & J_48 & 99 \\
\hline & & & CTC & 97 \\
\hline & & & J48graft & 98.75 \\
\hline & & & LMT & 98 \\
\hline & & & NB_Tree & 98.5 \\
\hline & & & Random_Forest & 100 \\
\hline & & & Random_Tree & 95.5 \\
\hline & & & REP Tree & 96.75 \\
\hline & & & Simple Cart & 97.5 \\
\hline Shetty, et al. (2019) & CKD Prediction & Pycharm & SVM & 90.09 \\
\hline Ahmad, M., et al. (2017) & $\begin{array}{l}\text { Chronic condition of } \\
\text { kidney disease }\end{array}$ & $\mathrm{R}$ & SVM & 98.34 \\
\hline \multirow{4}{*}{ Alassaf, R.A., et al. (2018) } & \multirow{4}{*}{$\begin{array}{l}\text { Preemptive Diagnosis } \\
\text { of Chronic Kidney } \\
\text { Disease }\end{array}$} & \multirow{4}{*}{ Weka and Python } & ANN & 98 \\
\hline & & & SVM & 98 \\
\hline & & & Naïve Bayes & 98 \\
\hline & & & K-NN & 93.9 \\
\hline \multirow{4}{*}{ Aljaaf, A.J., et al. (2018) } & \multirow{4}{*}{ Initial Estimate of CKD } & \multirow{4}{*}{ MATLAB } & RPART & 95.6 \\
\hline & & & SVM & 95 \\
\hline & & & LOGR & 98.1 \\
\hline & & & MLP & 98.1 \\
\hline \multirow{2}{*}{$\begin{array}{l}\text { Arif-Ul-Islam and Ripon, S.H., } \\
\text { Rule (2019) }\end{array}$} & \multirow{2}{*}{$\begin{array}{l}\text { Regulation Orientation } \\
\text { of CKD }\end{array}$} & \multirow{2}{*}{ Weka } & AdaBoost & 99 \\
\hline & & & LogitBoost & 99.75 \\
\hline \multirow{4}{*}{ Avci, E., et al. (2018) } & \multirow{4}{*}{$\begin{array}{l}\text { Performance } \\
\text { comparison of CKD }\end{array}$} & \multirow{4}{*}{ WEKA } & NB & 95 \\
\hline & & & k-star & 97.75 \\
\hline & & & SVM & 91.75 \\
\hline & & & $\mathrm{J} 48$ & 99 \\
\hline
\end{tabular}


Table 1. Continued

\begin{tabular}{|c|c|c|c|c|}
\hline Author & Problem & Software & Techniques & $\begin{array}{c}\text { Accuracy } \\
\text { Performance }(\%)\end{array}$ \\
\hline \multirow{3}{*}{ Banerjee, A., et al. (2019) } & \multirow{3}{*}{$\begin{array}{l}\text { Food Recommendation } \\
\text { of CKD }\end{array}$} & \multirow{3}{*}{ MATLAB } & Random Forest & 99.75 \\
\hline & & & SVM & 98.25 \\
\hline & & & Naïve Bayes & 95.5 \\
\hline \multirow{4}{*}{$\begin{array}{l}\text { Basarslan, M.S., and Kayaalp, } \\
\text { F., (2019) }\end{array}$} & \multirow{4}{*}{$\begin{array}{l}\text { Detection of Chronic } \\
\text { Kidney Disease }\end{array}$} & \multirow{4}{*}{ MATLAB } & K-Nearest_Neighbor & 97 \\
\hline & & & Navie_Bayes & 96.5 \\
\hline & & & LR & 97.56 \\
\hline & & & RF & 99 \\
\hline $\begin{array}{l}\text { Bhaskar, N., and Suchetha, } \\
\text { M., (2019) }\end{array}$ & $\begin{array}{l}\text { Automated Sensing } \\
\text { of Chronic Kidney } \\
\text { Disease }\end{array}$ & MATLAB & CNN-SVM & 98.04 \\
\hline \multirow{2}{*}{ Lakshmanaprabu, S.K., et al. } & \multirow{2}{*}{$\begin{array}{l}\text { Clinical decision } \\
\text { support system }\end{array}$} & \multirow{2}{*}{ MATLAB } & DNN & 98.25 \\
\hline & & & PSO-DNN & 99.25 \\
\hline \multirow{5}{*}{ Shankar, K., et al. (2018) } & \multirow{5}{*}{$\begin{array}{l}\text { Finest attribute } \\
\text { Selection }\end{array}$} & \multirow{5}{*}{ MATLAB } & D_N_N & 98 \\
\hline & & & C_N_N & 90 \\
\hline & & & N_N & 92 \\
\hline & & & B_P & 80 \\
\hline & & & K_N_N & 79 \\
\hline \multirow{2}{*}{ Zhang, H., et al. (2018) } & \multirow{2}{*}{ Survival Prediction } & \multirow{2}{*}{ Python } & Classical MLPs & 97.69 \\
\hline & & & LASSO preset MLPs & 93.23 \\
\hline \multirow{2}{*}{$\begin{array}{l}\text { Dulhare, U.N., and } \\
\text { Mohammad Ayesha, M., } \\
\text { (2016) }\end{array}$} & \multirow{2}{*}{ Mining of CKD } & \multirow{2}{*}{ Weka } & Naïve Bayes & 85 \\
\hline & & & Naïve Bayes with OneR & 97.5 \\
\hline \multirow{3}{*}{ Devika R, et al. (2019) } & \multirow{3}{*}{ Classify to CKD } & \multirow{3}{*}{ MATLAB } & Naive_Bayes & 99.635 \\
\hline & & & K_N_N & 87.78 \\
\hline & & & Random_Forest & 99.844 \\
\hline \multirow{2}{*}{ Jain, D., and Singh, V., (2018) } & \multirow{2}{*}{ Various level of CKD } & \multirow{2}{*}{ MATLAB } & SVM & 99 \\
\hline & & & ANN & 95 \\
\hline $\begin{array}{l}\text { Lee, M.-C., Wu, S.-F. V., } \\
\text { Hsieh, N.-C., \& Tsai, J.-M. } \\
\text { (2016) }\end{array}$ & $\begin{array}{l}\text { Kidney_Self-esteemed } \\
\text { progress }\end{array}$ & Meta- data-Analysis & - & - \\
\hline \multirow{2}{*}{ Almansour, N.A., et al. (2019) } & \multirow{2}{*}{$\begin{array}{l}\text { NN and SVM } \\
\text { prediction CKD: } \\
\text { Review study }\end{array}$} & \multirow{2}{*}{ WEKA } & ANN & 99.75 \\
\hline & & & SVM & 97.75 \\
\hline \multirow{6}{*}{ Zhao, J., et al. (2019) } & \multirow{6}{*}{$\begin{array}{l}\text { Expecting } \\
\text { consequences of CKD } \\
\text { using EMR }\end{array}$} & \multirow{6}{*}{ MATLAB } & Random Forest & 98.75 \\
\hline & & & $\begin{array}{l}\text { Sequential Minimal } \\
\text { Optimization, }\end{array}$ & 97.75 \\
\hline & & & Naïve Bayes & 98.25 \\
\hline & & & Radial Basis Function & 95 \\
\hline & & & $\begin{array}{l}\text { Multilayer Perceptron } \\
\text { Classifier }\end{array}$ & 90 \\
\hline & & & Simple Logistic & 92 \\
\hline
\end{tabular}


Figure 2. CKD Dataset Loading $\mathbf{R}$

\begin{tabular}{|c|c|c|c|c|c|c|c|c|c|c|c|c|c|c|c|c|c|c|c|c|c|}
\hline & ageg & sex & sbp & $d b p$ & hen & smoking & alc & sug & rbc & pce11 & pecellc & bac & bax & blu & sercr & sdi & pota & hg & DCV & wbec & rbec \\
\hline 1 & 3 & 2 & 150 & 70 & 2 & 0 & 0 & 0 & normal & normal & notpresent & notpresent & 121 & 36.0 & 1.20 & 145 & 4.2 & 15.4 & 44 & 7800 & 5.2 \\
\hline 2 & 2 & 2 & 110 & 70 & 1 & 0 & 0 & 0 & normal & abnormal & notpresent & notpresent & 128 & 36.0 & 1.20 & 141 & 4.4 & 15.4 & 44 & 7800 & 5.2 \\
\hline 3 & 2 & 2 & 150 & 80 & 2 & 0 & 0 & 0 & normal & normal & notpresent & notpresent & 100 & 22.0 & 0.70 & 136 & 4.8 & 10.7 & 34 & 12300 & 5.2 \\
\hline 4 & 3 & 2 & 130 & 80 & 1 & 0 & 0 & 0 & normal & normal & notpresent & notpresent & 99 & 23.0 & 0.60 & 138 & 4.4 & 12 & 34 & 12300 & 5.2 \\
\hline 5 & 4 & 2 & 130 & 90 & 2 & 0 & 0 & 0 & normal & normal & notpresent & notpresent & 65 & 16.0 & 0.70 & 138 & 3.2 & 8.1 & 34 & 12300 & 5.2 \\
\hline 6 & 4 & 2 & 150 & 70 & 2 & 0 & 0 & 0 & normal & normal & notpresent & notpresent & 83 & 25.0 & 0.60 & 138 & 3.2 & 11.8 & 36 & 12400 & 5.2 \\
\hline 7 & 4 & 2 & 120 & 70 & 1 & 0 & 0 & 0 & abnormal & abnormal & notpresent & present & 94 & 67.0 & 1.00 & 135 & 4.9 & 9.9 & 30 & 16700 & 4.8 \\
\hline 8 & 3 & 2 & 140 & 70 & 2 & 0 & 0 & 0 & abnormal & normal & notpresent & notpresent & 89 & 18.0 & 0.80 & 135 & 4.9 & 11.3 & 38 & 6000 & 4.8 \\
\hline 9 & 4 & 2 & 120 & 70 & 1 & 0 & 0 & 0 & normal & normal & notpresent & notpresent & 78 & 27.0 & 0.90 & 135 & 4.9 & 12.3 & 41 & 6700 & 4.8 \\
\hline 10 & 3 & 2 & 145 & 70 & 2 & 0 & 0 & 0 & normal & normal & notpresent & notpresent & 72 & 46.0 & 1.00 & 135 & 3.8 & 12.3 & 41 & 6700 & 4.8 \\
\hline 11 & 2 & 2 & 130 & 80 & 1 & 0 & 0 & 0 & normal & normal & notpresent & notpresent & 80 & 66.0 & 2.50 & 142 & 3.6 & 12.2 & 38 & 6700 & 4.8 \\
\hline 12 & 3 & 1 & 130 & 90 & 2 & 0 & 0 & 0 & normal & normal & notpresent & notpresent & 86 & 17.0 & 0.80 & 142 & 3.6 & 15 & 45 & 8600 & 4.8 \\
\hline 13 & 2 & 1 & 100 & 90 & 2 & 1 & 1 & 0 & abnorma 1 & abnormal & present & notpresent & 65 & 51.0 & 1.80 & 142 & 3.6 & 12.1 & 45 & 10300 & 4.8 \\
\hline 14 & 2 & 1 & 140 & 70 & 2 & 0 & 0 & 0 & normal & norma1 & notpresent & notpresent & 100 & 26.0 & 0.60 & 137 & 4.4 & 15.8 & 49 & 6600 & 4.8 \\
\hline 15 & 3 & 2 & 130 & 80 & 1 & 0 & 0 & 0 & normal & normal & notpresent & notpresent & 192 & 15.0 & 0.80 & 137 & 4.2 & 14.3 & 40 & 9500 & 5.4 \\
\hline 16 & 4 & 2 & 150 & 70 & 2 & 0 & 0 & 0 & normal & norma 1 & notpresent & notpresent & 86 & 15.0 & 0.60 & 138 & 4.0 & 11 & 33 & 7700 & 3.8 \\
\hline 17 & 4 & 2 & 130 & 90 & 2 & 0 & 0 & 0 & normal & normal & notpresent & notpresent & 93 & 17.0 & 0.90 & 136 & 3.9 & 16.7 & 50 & 6200 & 5.2 \\
\hline 18 & 3 & 2 & 130 & 90 & 2 & 0 & 0 & 0 & normal & normal 1 & notpresent & notpresent & 92 & 32.0 & 2.10 & 141 & 4.2 & 13.9 & 52 & 7000 & 5.2 \\
\hline 19 & 3 & 2 & 150 & 70 & 2 & 0 & 0 & 0 & abnorma1 & normal 1 & notpresent & notpresent & 22 & 1.5 & 7.30 & 145 & 2.8 & 13.1 & 41 & 11200 & 5.2 \\
\hline 20 & 3 & 2 & 110 & 90 & 2 & 0 & 0 & 0 & norma1 & normal & notpresent & notpresent & 114 & 50.0 & 1.00 & 135 & 4.9 & 14.2 & 51 & 7200 & 5.9 \\
\hline 21 & 3 & 1 & 130 & 80 & 1 & 0 & 0 & 0 & norma1 & norma 1 & notpresent & notpresent & 114 & 50.0 & 1.00 & 135 & 4.9 & 11.5 & 51 & 6900 & 5.9 \\
\hline 22 & 4 & 2 & 130 & 70 & 1 & 0 & 0 & 0 & norma1 & norma 1 & notpresent & notpresent & 107 & 23.0 & 0.70 & 141 & 4.2 & 14.4 & 44 & 6900 & 5.9 \\
\hline 23 & 4 & 2 & 140 & 70 & 2 & 0 & 0 & 0 & normal & norma 1 & notpresent & notpresent & 107 & 23.0 & 0.70 & 137 & 4.7 & 14 & 41 & 4500 & 5.5 \\
\hline 24 & 1 & 2 & 150 & 70 & 2 & 0 & 0 & 0 & norma1 & norma 1 & notpresent & notpresent & 123 & 44.0 & 1.00 & 135 & 3.8 & 14.6 & 44 & 5500 & 4.8 \\
\hline 25 & 4 & 2 & 150 & 70 & 2 & 0 & 0 & 0 & normal & normal 1 & notpresent & notpresent & 123 & 44.0 & 1.00 & 135 & 3.8 & 14.6 & 44 & 5500 & 4.8 \\
\hline 26 & 2 & 2 & 150 & 70 & 2 & 0 & 0 & 0 & normal & abnormal & present & present & 107 & 40.0 & 1.70 & 125 & 3.5 & 8.3 & 23 & 12400 & 3.9 \\
\hline 27 & 3 & 2 & 150 & 70 & 2 & 0 & 0 & 0 & normal & normal & notpresent & notpresent & 97 & 18.0 & 1.20 & 198 & 4.3 & 13.5 & 42 & 7900 & 6.4 \\
\hline 28 & 4 & 2 & 150 & 70 & 2 & 0 & 0 & 0 & normal & normal & notpresent & notpresent & 70 & 36.0 & 1.00 & 150 & 4.6 & 17 & 52 & 9800 & 5.0 \\
\hline 29 & 2 & 2 & 150 & 70 & 2 & 0 & 0 & 0 & normal & normal & notpresent & notpresent & 111 & 34.0 & 1.10 & 145 & 4.0 & 14.3 & 41 & 7200 & 5.0 \\
\hline 30 & 1 & 2 & 125 & 82 & 1 & 0 & 0 & 0 & normal & normal & notpresent & notpresent & 99 & 46.0 & 1.20 & 142 & 4.0 & 17.7 & 46 & 4300 & 5.5 \\
\hline
\end{tabular}

Figure 3. Finally, feature selection attributes only selected to $\mathbf{R}$

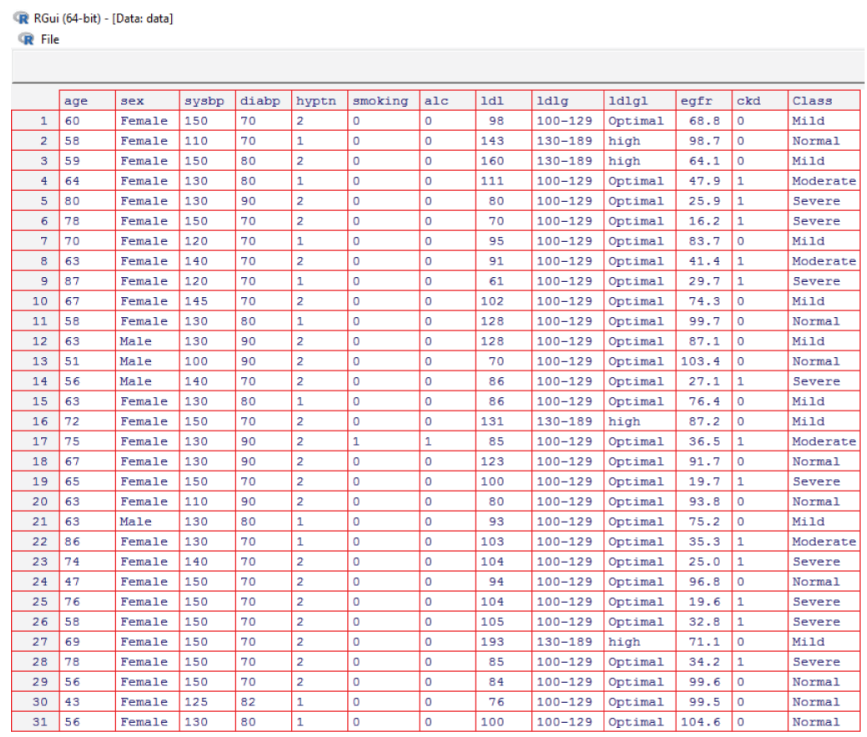

Entropy $\left(\mathrm{D}_{\mathrm{j}}\right)=-\sum_{j=1}^{m} p j \log 2(p j)$

$\operatorname{Info}$ Gain $(\mathrm{D}, \mathrm{A})=\operatorname{Entropy}\left(\mathrm{D}_{\mathrm{j}}\right)-\sum_{j=1}^{v} \frac{D j}{D} * \operatorname{Entropy}(D j)$

Gain Ratio (A) = Entropy (D) - Information Gain ${ }_{\mathrm{A}}(\mathrm{D})$ 
Figure 4. OneR

$\begin{array}{lr} & \text { attr_importance } \\ \text { ID } & 0.000000 \mathrm{e}+00 \\ \text { age } & 2.148148 \mathrm{e}-02 \\ \text { ageg } & 1.481481 \mathrm{e}-02 \\ \text { sex } & 5.551115 \mathrm{e}-17 \\ \text { occu3 } & 5.551115 \mathrm{e}-17 \\ \text { edu2 } & 5.551115 \mathrm{e}-17 \\ \text { sbp } & 0.000000 \mathrm{e}+00 \\ \text { dbp } & 2.469136 \mathrm{e}-03 \\ \text { htn } & 5.551115 \mathrm{e}-17 \\ \text { Ismoking } & 5.551115 \mathrm{e}-17 \\ \text { alc } & 5.551115 \mathrm{e}-17 \\ \text { dx } & 5.551115 \mathrm{e}-17 \\ \text { ldl } & 5.551115 \mathrm{e}-17 \\ \text { ldlg } & 5.551115 \mathrm{e}-17 \\ \text { uhe } & 5.551115 \mathrm{e}-17 \\ \text { egfr } & 2.535802 \mathrm{e}-01\end{array}$

Figure 5. Random

$\begin{array}{lr}\text { ID } & 5.4184276 \\ \text { age } & 12.0455084 \\ \text { ageg } & 8.5505324 \\ \text { sex } & 0.3803352 \\ \text { occu3 } & 2.3442413 \\ \text { edu2 } & -1.9016220 \\ \text { sbp } & 8.6162261 \\ \text { dbp } & 3.5450150 \\ \text { htn } & 7.3814191 \\ \text { smoking } & -0.4258979 \\ \text { alc } & -0.3421356 \\ \text { dx } & 6.7957406 \\ \text { ldl } & 4.9443505 \\ \text { ldlg } & 4.6541099 \\ \text { uhc } & -0.7720223 \\ \text { egfr } & 422.1423430\end{array}$

Figure 6. Relief

$\begin{array}{lr} & \text { attr_importance } \\ \text { ID } & 2.600980 \mathrm{e}-02 \\ \text { age } & 2.897436 \mathrm{e}-02 \\ \text { ageg } & 7.666667 \mathrm{e}-02 \\ \text { sex } & 0.000000 \mathrm{e}+00 \\ \text { occu3 } & 2.500000 \mathrm{e}-02 \\ \text { edu2 } & 3.008081 \mathrm{e}-02 \\ \text { sbp } & 7.105263 \mathrm{e}-03 \\ \text { dbp } & 5.000000 \mathrm{e}-02 \\ \text { htn } & 0.000000 \mathrm{e}+00 \\ \text { smoking } & 0.000000 \mathrm{e}+00 \\ \text { alc } & 0.000000 \mathrm{e}+00 \\ \text { dx } & 0.000000 \mathrm{e}+00 \\ \text { ldl } & 6.321321 \mathrm{e}-03 \\ \text { ldlg } & 3.000000 \mathrm{e}-02 \\ \text { uhc } & -5.551115 \mathrm{e}-18 \\ \text { egfr } & 2.209169 \mathrm{e}-01\end{array}$


Figure 7. Symmetrical Uncertainty

$\begin{array}{lr} & \text { attr_importance } \\ \text { ID } & 0.017636518 \\ \text { age } & 0.092612490 \\ \text { ageg } & 0.087739999 \\ \text { sex } & 0.000000000 \\ \text { occu3 } & 0.029974558 \\ \text { edu2 } & 0.000000000 \\ \text { sbp } & 0.024272977 \\ \text { dbp } & 0.008686779 \\ \text { htn } & 0.024815333 \\ \text { smoking } & 0.000000000 \\ \text { alc } & 0.000000000 \\ \text { dx } & 0.024815333 \\ \text { ldl } & 0.000000000 \\ \text { ldlg } & 0.000000000 \\ \text { uhc } & 0.000000000 \\ \text { egfr } & 1.000000000\end{array}$

Figure 8. Gain Ratio

\begin{tabular}{|lr} 
& attr_importance \\
ID & 0.014043224 \\
age & 0.065389964 \\
ageg & 0.062190510 \\
sex & 0.000000000 \\
occu3 & 0.024307074 \\
edu2 & 0.000000000 \\
sbp & 0.022136135 \\
dbp & 0.006094753 \\
htn & 0.022700592 \\
smoking & 0.000000000 \\
alc & 0.000000000 \\
dx & 0.022700592 \\
ldl & 0.000000000 \\
ldlg & 0.000000000 \\
uhe & 0.000000000 \\
egfr & 1.000000000
\end{tabular}

Information Gain $_{\mathrm{A}}(\mathrm{D})=\sum_{j=1}^{v} \frac{D j}{D} * \operatorname{Entropy}(D j)$

This research work focuses on the gain ratio feature selection that comes under the filter method, which uses the measures entropy, information gain and gains ratio. Other feature selection methods are:

- Chi-square

- Random forest

- Relief

- OneR

- Symmetrical uncertainty 


\begin{tabular}{|lr} 
& attr_importance \\
ID & 0.1683307 \\
age & 0.4172747 \\
ageg & 0.4035741 \\
sex & 0.0000000 \\
occu3 & 0.2059137 \\
edu2 & 0.0000000 \\
sbp & 0.1720889 \\
dbp & 0.1255351 \\
htn & 0.1731719 \\
smoking & 0.0000000 \\
alc & 0.0000000 \\
dx & 0.1731719 \\
ldl & 0.0000000 \\
ldlg & 0.0000000 \\
uhc & 0.0000000 \\
egfr & 1.0000000
\end{tabular}

The third objective is the optimization of classical machine learning algorithms using another machine learning approaches to achieve high accuracy.

Part 3: Classification

- Decision Tree

- C4.5 Algorithm

- C5.0 Algorithm

Figure 10 represents the various parts are represented the patient data is evaluated with the healthcare data analytics block diagram of Chronic Kidney Disease (CKD).

\section{DATA COLLECTION}

The chronic kidney data set files are composed of prediction is based on the given attributes. This dataset has thirty-two attributes that predict the CKD. It contains an attribute such as age, age group sex, (systolic and diastolic) blood_pressure, specific_gravity, albumin, sugar, red_blood_cells, plus_cell, pus_cell_clumps, bacteria, blood_glucose_random, blood_urea, serum_creatine, sodium, potassium, hemoglobin, packed_cell_volume, white_blood_cell_count, red_blood_cell count, hypertension, diabetes_mellitus, appetite, pedal_edema, Low_density lipoprotein, smoking_status, alcohol_drinking, anemia, Estimated_glomerular_filtration_rate, CKD Level and Class. Initially, data size are 4050 records and 33 attributes are preprocessing, attribute variety techniques, cataloging or classification algorithms toward spread over chronic kidney data using performance evaluation (Tables 2-3).

\section{RESULTS}

\section{C5.0 Algorithm}

It is an important classification algorithm for decision tree. These algorithms are handling continued values or categorical values. Feature selection is an essential phase to create the 


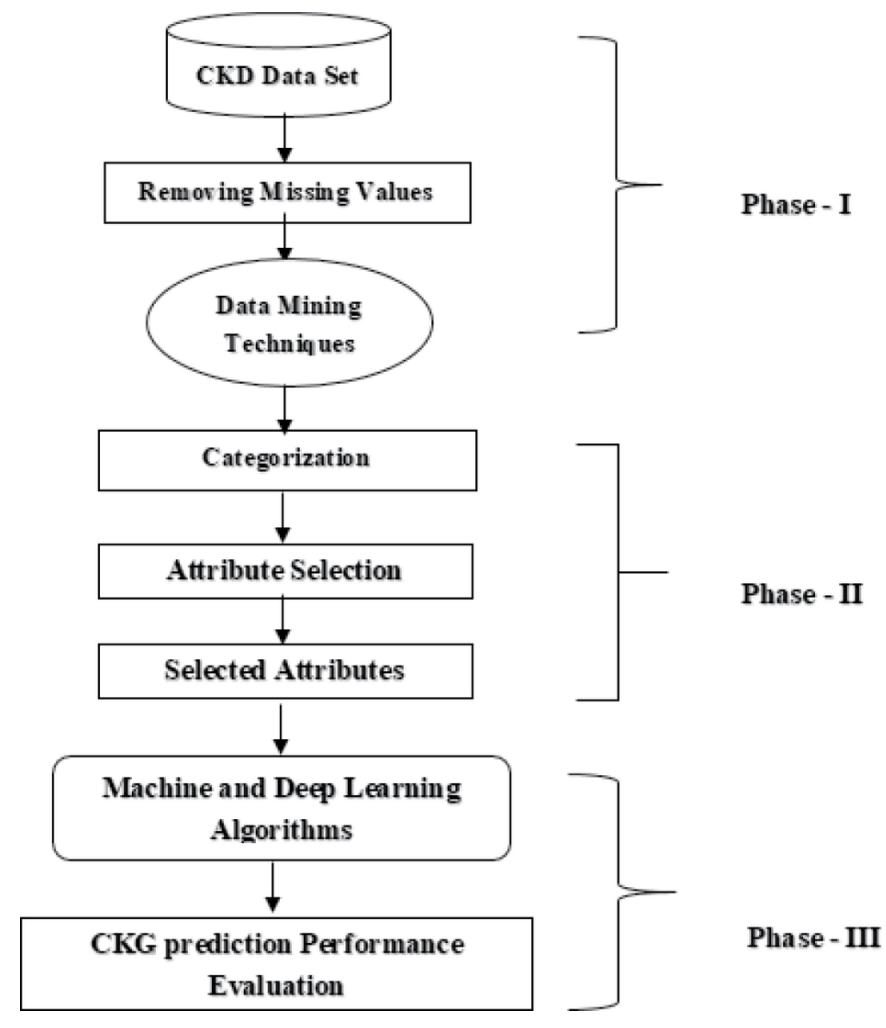

decision_tree. Associating to a Decision tree, C4.5 and C5.0 uppermost process. It was performed on pre_pruning. One of the decision tree classification algorithms. Entropy, information_gain and gain_ratio measures are considered and the classification model was developed. Applied on the data set to determine the unknown samples. Many classification methods are available to predict overall performance. That C5.0 is one of the best decision tree classification algorithms. It can handle continuous and categorical values. It can handle numeric attributes. Comparing with ID3, C4.5 and C5.0. C5.0 has the highest speed and pre-pruning. So, the proposed system carries out the prediction operation using the C5.0 classifier. Attribute selection is the fundamental step to construct a decision tree. Entropy, information gain, and gain ratio are used to process attribute selection. During attribute selection, C5.0 algorithm selects the root node of the decision tree (Figures 11-12).

Advantages:

- Accurate result

- Less memory space for the large data set

- Less time to build a model

- Increasing level support

- Highest speed

- Handle continuous value, categorical values and multi-value

Disadvantage:

- Empty branches and insignificant branches are allowed 
Table 2. Attributes of Chronic Kidney Disease Dataset

\begin{tabular}{|c|c|c|c|c|}
\hline S. No & Attribute Name & $\begin{array}{c}\text { Attribute } \\
\text { Type }\end{array}$ & $\begin{array}{l}\text { Attribute } \\
\text { Code }\end{array}$ & Possible Values \\
\hline 1. & Age & Numeric & age & $E, V G, G, F, P$ \\
\hline 2. & Age Group & Numeric & ageg & $\mathrm{E}, \mathrm{VG}, \mathrm{G}, \mathrm{F}, \mathrm{P}$ \\
\hline 3. & Sex & Nominal & Sex & $\mathrm{E}, \mathrm{VG}, \mathrm{G}, \mathrm{F}, \mathrm{P}$ \\
\hline 4. & Systolic Blood Pressure & Numeric & sysbp & $E, V G, G, F, P$ \\
\hline 5. & Diastolic Blood Pressure & Numeric & diabp & $\mathrm{E}, \mathrm{VG}, \mathrm{G}, \mathrm{F}, \mathrm{P}$ \\
\hline 6. & Specific Gravity & Numeric & sap & $\mathrm{E}, \mathrm{VG}, \mathrm{G}, \mathrm{F}, \mathrm{P}$ \\
\hline 7. & Albumin & Numeric & alb & E, VG, G, F, P \\
\hline 8. & Sugar & Numeric & sug & $\mathrm{E}, \mathrm{VG}, \mathrm{G}, \mathrm{F}, \mathrm{P}$ \\
\hline 9. & Red Blood Cell & Nominal & $\mathrm{rbc}$ & $E, V G, G, F, P$ \\
\hline 10. & Pus Cell & Nominal & pcell & $\mathrm{E}, \mathrm{VG}, \mathrm{G}, \mathrm{F}, \mathrm{P}$ \\
\hline 11. & Pus Cell Clumps & Nominal & pcellc & $\mathrm{E}, \mathrm{VG}, \mathrm{G}, \mathrm{F}, \mathrm{P}$ \\
\hline 12. & Bacteria & Numeric & $\mathrm{bac}$ & $\mathrm{E}, \mathrm{VG}, \mathrm{G}, \mathrm{F}, \mathrm{P}$ \\
\hline 13. & Blood Glucose Random & Numeric & bgr & $\mathrm{E}, \mathrm{VG}, \mathrm{G}, \mathrm{F}, \mathrm{P}$ \\
\hline 14. & Blood Urea & Numeric & blu & $\mathrm{E}, \mathrm{VG}, \mathrm{G}, \mathrm{F}, \mathrm{P}$ \\
\hline 15. & Serum Creatine & Numeric & sercr & $\mathrm{E}, \mathrm{VG}, \mathrm{G}, \mathrm{F}, \mathrm{P}$ \\
\hline 16. & Sodium & Numeric & sdi & $\mathrm{E}, \mathrm{VG}, \mathrm{G}, \mathrm{F}, \mathrm{P}$ \\
\hline 17. & Potassium & Numeric & pota & $E, V G, G, F, P$ \\
\hline 18. & Hemoglobin & Numeric & hg & $\mathrm{E}, \mathrm{VG}, \mathrm{G}, \mathrm{F}, \mathrm{P}$ \\
\hline 19. & Packed_Cell_Volume & Numeric & p_c_v & $\mathrm{E}, \mathrm{VG}, \mathrm{G}, \mathrm{F}, \mathrm{P}$ \\
\hline 20. & White_Blood_Cell_Count & Numeric & w_b_c_c & $\mathrm{E}, \mathrm{VG}, \mathrm{G}, \mathrm{F}, \mathrm{P}$ \\
\hline 21. & Red_Blood_Cell_Count & Numeric & r_b_c_c & $\mathrm{E}, \mathrm{VG}, \mathrm{G}, \mathrm{F}, \mathrm{P}$ \\
\hline 22. & Hypertension & Nominal & hyptn & $E, V G, G, F, P$ \\
\hline 23. & Diabetes Mellitus & Numeric & diam & $\mathrm{E}, \mathrm{VG}, \mathrm{G}, \mathrm{F}, \mathrm{P}$ \\
\hline 24. & Appetite & Nominal & app & $\mathrm{E}, \mathrm{VG}, \mathrm{G}, \mathrm{F}, \mathrm{P}$ \\
\hline 25. & Pedal Edema & Nominal & peed & $\mathrm{E}, \mathrm{VG}, \mathrm{G}, \mathrm{F}, \mathrm{P}$ \\
\hline 26. & Low Density Lipoprotein & Numeric & ldl & $\mathrm{E}, \mathrm{VG}, \mathrm{G}, \mathrm{F}, \mathrm{P}$ \\
\hline 27. & smoking status & Numeric & smo & $\mathrm{E}, \mathrm{VG}, \mathrm{G}, \mathrm{F}, \mathrm{P}$ \\
\hline 28. & Alcohol Drinking & Numeric & alc & $\mathrm{E}, \mathrm{VG}, \mathrm{G}, \mathrm{F}, \mathrm{P}$ \\
\hline 29. & Anemia & Nominal & ane & E, VG, G, F, P \\
\hline 30. & Coronary Artery Disease & Nominal & Coad & $\mathrm{E}, \mathrm{VG}, \mathrm{G}, \mathrm{F}, \mathrm{P}$ \\
\hline 31. & Estimated Glomerular Filtration Rate & Numeric & egfr & $\mathrm{E}, \mathrm{VG}, \mathrm{G}, \mathrm{F}, \mathrm{P}$ \\
\hline 32. & CKD Level & $\begin{array}{l}\text { Numeric or } \\
\text { Nominal }\end{array}$ & ckd & $\mathrm{E}, \mathrm{VG}, \mathrm{G}, \mathrm{F}, \mathrm{P}$ \\
\hline 33. & Class & $\begin{array}{l}\text { Numeric or } \\
\text { Nominal }\end{array}$ & Class & $\mathrm{E}, \mathrm{VG}, \mathrm{G}, \mathrm{F}, \mathrm{P}$ \\
\hline
\end{tabular}


Table 3. Testing Performance for Chronic Kidney Disease Identification

\begin{tabular}{|l|l|l|}
\hline \multicolumn{1}{|c|}{ Main Testing } & \multicolumn{2}{|c|}{ Prediction } \\
\hline \multirow{4}{*}{$\begin{array}{l}\text { All attributes measure level compare to Estimated Glomerular } \\
\text { Filtration Rate value(egfr) }\end{array}$} & Excellent & Normal \\
\cline { 2 - 3 } & Very Good & Mild \\
\cline { 2 - 3 } & Good & Moderate \\
\cline { 2 - 3 } & Fair & Severe \\
\cline { 2 - 3 } & Poor or Failure & End-stage \\
\hline
\end{tabular}

Figure 11. C5.0 Algorithm

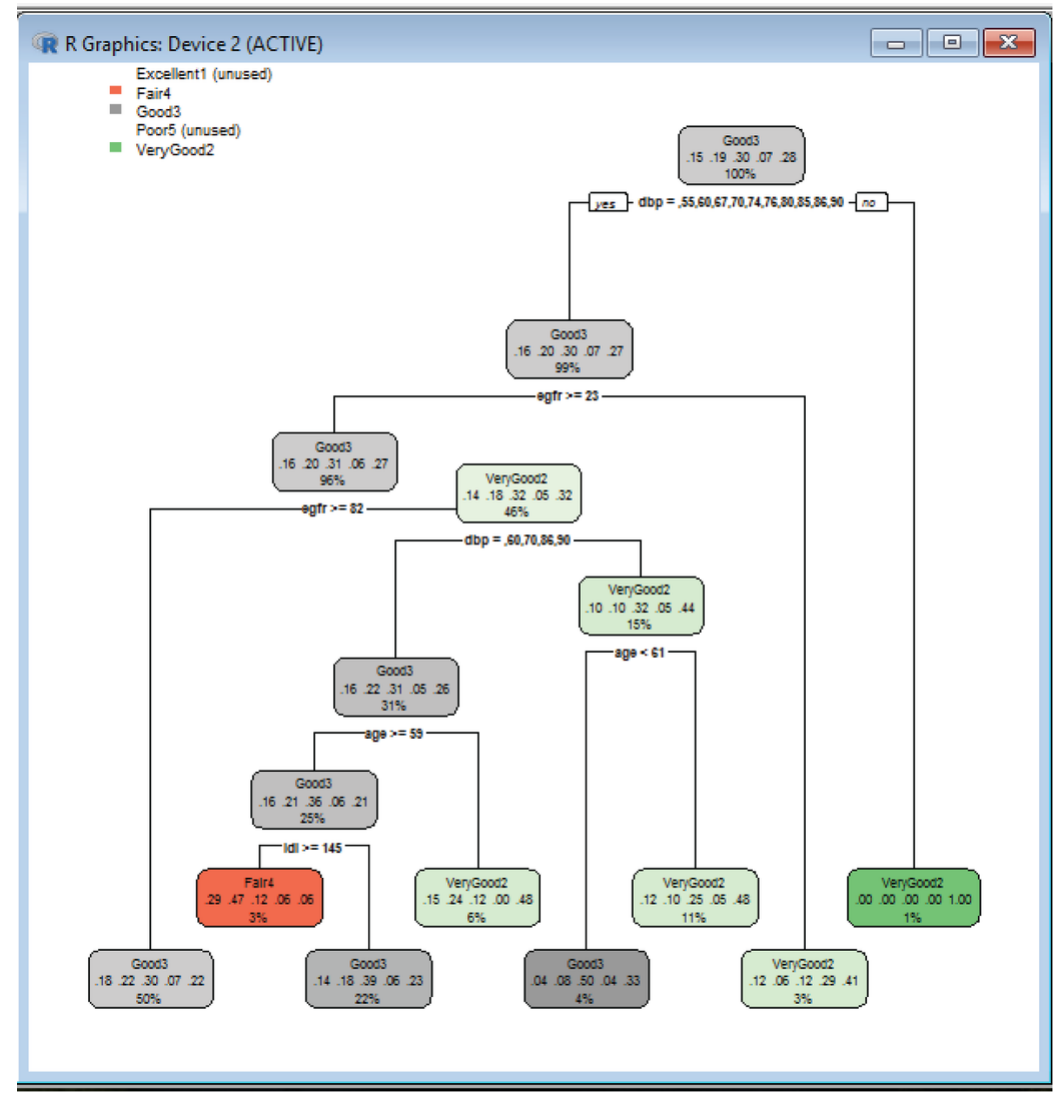

Figure 12. Pseudocode for C5.0 Classifier

Input: Chronic Kidney Data
Output: Selected Attributes
Step1: Read the data
Step2: Calculate entropy value
Step3: Compute information gain for each and every attribute
Step4: Compute gain ratio using entropy and information gain
Step5: Find the attribute with the highest gain ratio value
Step6: If there is no more attribute, the tree construction was
completed. (Returns a leaf labeled with the most frequent class
or the disjunction of all the classes).




\section{C5.0_Classification}

Numerous classification algorithms available to predict early stages are identified for CKD. It is the topmost algorithms. So, the proposed system carries out the prediction operation using the C5.0 classifier. In the proposed system, the C5.0 classifier classifies chronic kidney disease stages into predetermined classes such as normal (Excellent), mild (very good), moderate (Good), severe (Fair) and end-stage (Poor or Failure).

C5.0 algorithm applies the chronic kidney disease data to predicting all stages and identifies early stages for analyzing less time consuming and higher accuracy comparing other machine learning algorithms.

\section{DECISION TREE}

It signifies a test node. It is used to organize an order by beginning at the root other than a leaf node (Quinlan, 1986).

A decision tree is a supervised classification, which predicts both the classifier and regression models. Classification trees are mainly used to classify an object to a predetermined class based on the attributes. The tree contains no incoming edge is called as root, The node through one outward edge is named an internal_node, all other nodes stay notorious as leaf node which has no outgoing edge. Using the training sets the classifier model has been developed, the testing set was applied to the classification model to predict the previously unknown class (Figure 13).

\section{C4.5 Algorithm}

C4.5 is the basic classification algorithm for decision tree. It was developed by Quinlan. To uses a gain_ratio by way of a split the selection process. By calculating entropy and splitting information of an attribute. It is based on attributes selection for numeric and missing data values. Faster than the ID3 algorithm. It also cannot deal with missing values. A decision tree is built to scrutinizing a regular training examples class brands are identified. This selection is an identified model smeared to decide the property of unidentified models (Figures 14-15).

Advantages:

- accurate result

- less memory space for the large data set

- less time to build a model

- short searching time

Disadvantages:

- Empty branches and insignificant branches are allowed

- Overfitting is one of the most important problems in the C4.5 algorithm

Figure 13. Pseudocode for Decision Tree

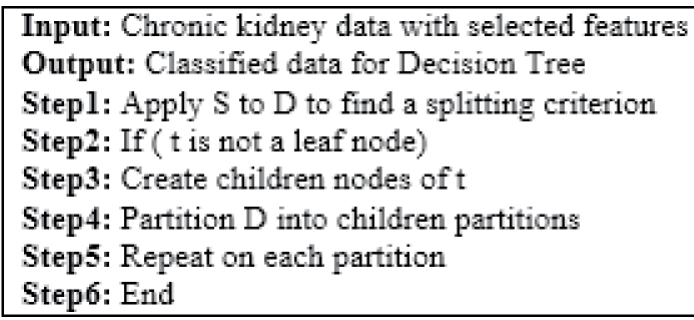


Figure 14. Pseudocode for C4.5 Algorithm

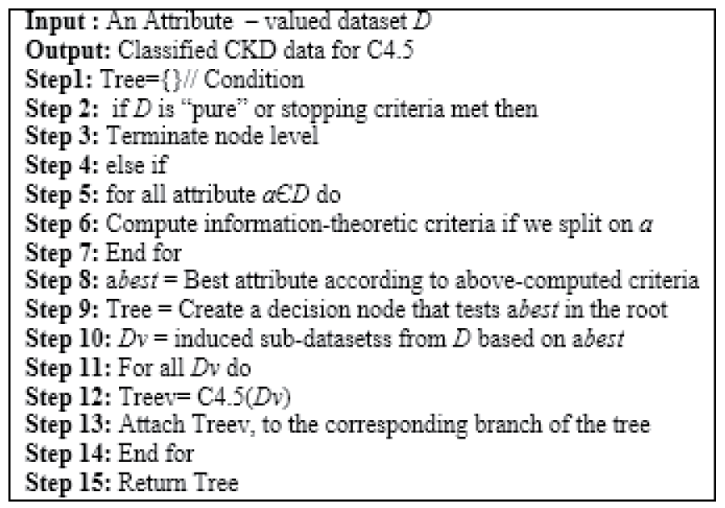

Figure 15. Accuracy performance using various machine learning algorithms

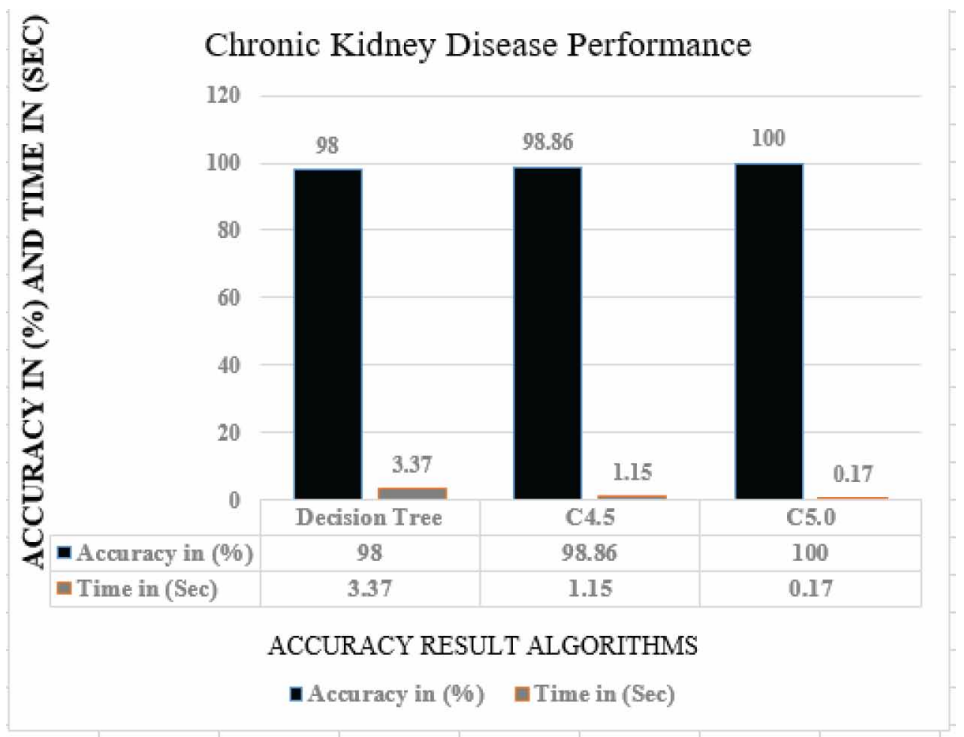

\section{CONCLUSION}

In conclusion, chronic kidney disease considers approaching toward developing recommendations for machine learning techniques in healthcare has become a real-world emerging for obtaining accurate results of medical diagnosis, using the machine learning techniques involved the healthcare is evolving into a promising field for improving outcomes with reducing costs. Thus the system can improve the efficiency of mining risk factors of chronic kidney disease, but there are also have some shortcomings. To overcome these issues, improve an effectual clinical judgment care structure chronic kidney disease decision support system consuming classification algorithms over a large volume of the dataset for making better decisions and predictions. The gain ratio feature selection method is best and fewer time associates other selection methods. The information is verified by classification C5.0 algorithms. Then to predict chronic kidney disease using the C5.0 is high accuracy bring about and less time complexity in 100\% cataloging accuracy. 


\section{REFERENCES}

Abeer, Y., \& Al-Hyari. (2012). Chronic kidney disease prediction system using classifying data mining techniques. Library of University of Jordan.

Ahmad, M., Tundjungsari, V., Widianti, D., Amalia, P., \& Rachmawati, U. A. (2017). Diagnostic Decision Support System of Chronic Kidney Disease Using Support Vector Machine. Second International Conference on Informatics and Computing (ICIC). doi:10.1109/IAC.2017.8280576

Alasker, H., Alharkan, S., Alharkan, W., \& Riza, L. S. (2017). Detection of Kidney Disease Using Various Intelligent Classifiers. 2017 3rd International Conference on Science in Information Technology (ICSITech), 681-684. doi:10.1109/ICSITech.2017.8257199

Alassaf, R. A., Alsulaim, K. A., Alroomi, N. Y., Alsharif, N. S., Aljubeir, M. F., Olatunji, S. O., Alahmadi, A. Y., Imran, M., Alzahrani, R. A., \& Alturayeif, N. S. (2018). Preemptive Diagnosis of Chronic Kidney Disease Using Machine Learning Techniques. 13 International Conferences on Innovations in Information Technology, 2018 IEEE, 99-104.

Aljaaf, A. J., Al-Jumeily, D., \& Hussein, M. (2018). Early Prediction of Chronic Kidney Disease Using Machine Learning Supported by Predictive Analytics. IEEE Congress on Evolutionary Computation (CEC).

Almansour, N. A., Syed, H. S., Khayat, N. R., Altheeb, R. K., Juri, R. E., Alhiyafi, J., Alrashed, S., \& Olatunji, S. O. (2019). Neural network and support vector machine for the prediction of chronic kidney disease: A comparative study. Computers in Biology and Medicine, 109, 101-111. doi:10.1016/j.compbiomed.2019.04.017 PMID:31054385

Arif-Ul-Islam, , \& Ripon, , S.H. (2019). Rule Induction and Prediction of Chronic Kidney Disease Using Boosting Classifiers, Ant-Miner and J48 Decision Tree. 2019 International Conference on Electrical, Computer and Communication Engineering (ECCE).

AVCI. (2018). Performance Comparison of Some Classifiers on Chronic Kidney Disease Data. IEEE.

Bala, S., \& Kumar, K. (2014, July). A Literature Review on Kidney Disease Prediction using Data Mining Classification Technique. IJCSMC, 3(7), 960-967.

Banerjee, A., Noor, A., Siddiqua, N., \& Uddin, M. N. (2019). Significance of Attribute Selection In The Classification of Chronic Renal Disease. Second International Conference on Advanced Computational and Communication Paradigms (ICACCP). doi:10.1109/ICACCP.2019.8882937

Basarslan, M. S., \& Kayaalp, F. (2019). Performance Analysis of Fuzzy Rough Set-Based and CorrelationBased Attribute Selection Methods on Detection of Chronic Kidney Disease with Various Classifiers. IEEE. doi:10.1109/EBBT.2019.8741688

Bhaskar, N., \& Suchetha, M. (2019). A Deep Learning-based System for Automated Sensing of Chronic Kidney Disease. IEEE Sensors Letters, 3(10).

Celik, E., Atalay, M., \& Kondiloglu, A. (2014). The Diagnosis and Estimate of Chronic Kidney Disease Using the Machine Learning Methods. International Journal of Intelligent Systems and Applications in Engineering, 4, 27-31.

Chatterjee, S., Dzitac, S., Sen, S., Rohatinovici, N. C., Dey, N., Ashour, A. S., \& Balas, V. E. (2017). Hybrid Modified Cuckoo Search-Neural Network in Chronic Kidney Disease Classification. 14th International Conference on Engineering of Modern Electric Systems (EMES). doi:10.1109/EMES.2017.7980405

Devika, R., Avilala, S. V., \& Subramaniyaswamy, V. (2019). Comparative Study of Classifier for Chronic Kidney Disease prediction using Naive Bayes, KNN and Random Forest. Proceedings of the Third International Conference on Computing Methodologies and Communication (ICCMC 2019). doi:10.1109/ICCMC.2019.8819654

Dowluru, K., Rayavarapu, A.K., \& Vadlapudi, V. (2012). Statistical and Data Mining Aspects on Kidney Stones: A Systematic Review and Meta-analysis. Open Access Scientific Reports, 1(12).

Dulhare, U. N., \& Mohammad Ayesha, M. (2016). Extraction of Action Rules for Chronic Kidney Disease using Naïve Bayes Classifier. IEEE International Conference on Computational Intelligence and Computing Research. doi:10.1109/ICCIC.2016.7919649 
Eyck, V. J., Ramon, J., Guiza, F., Meyfroidt, G., Bruynooghe, M., \& Berghe, V. G. (2012). Data mining techniques for predicting acute kidney injury after elective cardiac surgery. Springer.

Feature Selection. (n.d.). In Wikipedia. https://en.wikipedia.org/wiki/Feature_selection

HKU. (n.d.). https://ar.cetl.hku.hk/am_literature_reviews.htm

Jain, D., \& Gautam, S. (2014). Predicting the Effect of Diabetes on Kidney using Classification in Tanagra. International Journal of Computer Science and Mobile Computing, 3(4).

Jain, D., \& Singh, V. (2018). Feature selection and classification systems for chronic disease prediction: A review. Egyptian Informatics Journal, 19, 179-189.

Jena, L., \& Kamila, N. K. (2015). Distributed Data Mining Classification Algorithms for Prediction of ChronicKidney-Disease. International Journal of Emerging Research in Management \&Technology, 4(11).

Jose, J.S., Sivakami, R., UmaMaheswari, N., \& Venkatesh, R. (2012). An Efficient Diagnosis of Kidney Images using Association Rules. International Journal of Computer Technology and Electronics Engineering, 2(2).

Kumar, K., \& Abhishek, . (2012). Artificial Neural Networks for Diagnosis of Kidney Stones Disease. I.J. Information Technology and Computer Science, 7, 20-25.

Kumar, M. (2016). Prediction of Chronic Kidney Disease Using Random Forest Machine Learning Algorithm. International Journal of Computer Science and Mobile Computing, 5(2), 24-33.

Lakshmanaprabu, S. K., Mohanty, S. N., Rani, S. S., Krishnamoorthy, S., Uthayakumar, J., \& Shankar, K. (2019). Online clinical decision support system using optimal deep neural Networks. Applied Soft Computing, 81, 105487. doi:10.1016/j.asoc.2019.105487

Lakshmi, K.R., Nagesh, Y., \& VeeraKrishna, M. (2014). Performance comparison of three data mining techniques for predicting kidney disease survivability. International Journal of Advances in Engineering \& Technology.

Lee, M.-C., Wu, S.-F. V., Hsieh, N.-C., \& Tsai, J.-M. (2016). Self-Management Programs on eGFR, Depression, and Quality of Life among Patients with Chronic Kidney Disease: A Meta-Analysis. Asian Nursing Research, 10(4), 255-262. doi:10.1016/j.anr.2016.04.002 PMID:28057311

Leung, R. K. K., Wang, Y., Ma, R. C. W., Luk, A. O. Y., Lam, V., Ng, M., So, W. Y., Tsui, S. K. W., \& Chan, J. C. N. (2013). Using a multi-staged strategy based on machine learning and mathematical modeling to predict genotype-phenotype risk patterns in diabetic kidney disease: A prospective case-control cohort analysis. BMC Nephrology, 14(1), 162. doi:10.1186/1471-2369-14-162 PMID:23879411

Mahdavi-mazdeh, M., Yadollahpour, A., Nourozi, J., Mirbagheri, S. A., Macotela, F. R. T., \& Simancas-Acevedo, E. (2018). Designing and implementing an ANFIS based medical decision support system to predict chronic kidney disease progression. Frontiers in Physiology. www.frontiersin.org

Norouzi, J., Yadollahpour, A., AhmadMirbagheri, S., Mazdeh, M., \& Hosseini, S.A. (2016). Predicting Renal Failure Progression in Chronic Kidney Disease Using Integrated Intelligent Fuzzy Expert System. Computational and Mathematical Methods in Medicine. 10.1155/2016/6080814

Pasadana, I. A., Hartama, D., Zarlis, M., \& Sianipar, A. S. (2019). Chronic Kidney Disease Prediction by Using Different Decision Tree Techniques. The International Conference on Computer Science and Applied Mathematics. doi:10.1088/1742-6596/1255/1/012024

Queens U. (n.d.). https://library.queensu.ca/webedu/grad/Purpose_of_the_Literature_Review.pdf

Quinlan, J. R. (1986). Induction of decision trees. Machine Learning, 1(1), 81-106. doi:10.1007/BF00116251

Ramya, S., \& Radha, N. (2016). Diagnosis of Chronic Kidney Disease Using Machine Learning Algorithms. International Journal of Innovative Research in Computer and Communication Engineering, 4(1). DOI: .0401049 81210.15680/IJIRCCE.2016

Reddy, C. K., \& Aggarwal, C. C. (2015). Healthcare Data Analytics. CRC Press Taylor \& Francis Group. doi:10.1201/b18588 
Shankar, K., Manickam, P., Devika, G., \& Ilayaraja, M. (2018). Optimal Feature Selection for Chronic Kidney Disease Classification using Deep Learning Classifier. IEEE International Conference on Computational Intelligence and Computing Research (ICCIC). doi:10.1109/ICCIC.2018.8782340

Sharma, S., Sharma, V., \& Sharma, A. (2016). Performance Based Evaluation of Various Machine Learning Classification Techniques for Chronic Kidney Disease Diagnosis. International Journal of Modern Computer Science, 4(3), 11-16.

Sheety, A. R., Ahmed, F. B., \& Naik, V. M. (2019). CDK prediction using Data Mining Techniques as SVM and KNN with Pycharm. International Research Journal of Engineering and Technology, 6(5).

Sinha, P., \& Sinha, P. (2015). Comparative Study of Chronic Kidney Disease Prediction using KNN and SVM. International Journal of Engineering Research \& Technology, 4(12). www.ijert.org

Song, X., Qiu, Z., \& Jianwei. (2012). Study on Data Mining Technology and its Application for Renal Failure Hemodialysis Medical Field. International Journal of Advancements in Computing Technology, 4(3).

Sriraam, N., Natashav, \& Kaur, H. (2006). Data mining approaches for kidney dialysis treatment. Journal of Mechanics in Medicine and Biology, 6(2).

Subhashini, R., \& Jeyakumar, M.K. (2017). Performance Analysis of Different Classification Techniques for the Prediction of Chronic Kidney Disease. International Journal of Pharmacy \& Technology, 9(4).

Urinary Incontinence. (n.d.). In WebMd. https://www.webmd.com/urinary-incontinence-oab

Vijayarani, S., \& Dhayanand, S. (2015a). Kidney Disease Prediction Using SVM And ANN Algorithms. International Journal of Computing and Business Research, 6(2).

Vijayarani, S., \& Dhayanand, S. (2015b, August). Data Mining Classification Algorithms for Kidney Disease Prediction. International Journal on Cybernetics \& Informatics, 4(4). Advance online publication. doi:10.5121/ ijci.2015.4402 13

Zadeh, M.K., Rezapour, M., \& Sepehri, M.M. (2013). Data Mining Performance in Identifying the Risk Factors of Early Arteriovenous Fistula Failure in Hemodialysis Patients. International Journal of Hospital Research, 2(1), 49-54.

Zhang, H., Hung, C., Chu, W. C., Chiu, P., \& Tang, C. Y. (2018). Chronic Kidney Disease Survival Prediction with Artificial Neural Networks. IEEE International Conference on Bioinformatics and Biomedicine (BIBM). doi:10.1109/BIBM.2018.8621294

Zhao, J., Gu, S., \& McDermaid, A. (2019). Predicting outcomes of chronic kidney disease from EMR data based on Random Forest Regression. Mathematical Biosciences, 310, 24-30. doi:10.1016/j.mbs.2019.02.001 PMID:30768948 